\title{
sciendo
}

\section{Differences in External Load Variables Between Playing Positions in Elite Basketball Match-Play}

\author{
by \\ Hugo Salazar ${ }^{1,2}$, Julen Castellano ${ }^{1}$, Luka Svilar ${ }^{2}$
}

\begin{abstract}
The purpose of this study was to describe the specific demands and structure of interrelationships of external load variables in order to generate a position-related time motion profile in elite basketball. Seventeen professional players from three different playing positions (6 guards, 4 forwards, and 7 centers) were analyzed in five friendly games. Player load per minute (PLmin) was used as an indicator of intensity to compare positions. Furthermore, high and total external variables of jumping ( $h J U M P$ and tJUMP), acceleration ( $h A C C$ and $t A C C$ ), deceleration ( $h D E C$ and $t D E C)$ and change of direction (hCOD and $t C O D)$, respectively, were used for the principal component analysis $(P C A)$. The Kaiser criterion (eigenvalue $>1$ ) was applied, and the Varimax rotation mode was used to extract multiple principal components. PCA showed that all positions had three or four principal components, but the configuration of each factor was different: $t C O D, h C O D, h D E C$ and hJUMP for guards, $h C O D, t C O D, t A C C$ and $h D E C$ for forwards, and tJUMP, hJUMP, hDEC and tACC for centers were specifically demanded in match-play. For guards and forwards, a significant correlation was found between COD variables, while for centers TCOD and PLmin had the strongest correlation. When monitoring the external load via tri-axial accelerometers in basketball match-play, each playing position showed specific physical demands. Therefore, these variables must be prioritized in load monitoring programs.
\end{abstract}

Key words: playing position, team sport, time motion, basketball, PCA, game load.

\section{Introduction}

In professional sports, the use of match performance analysis helps coaches investigate and analyze team and players' activities for the purpose of enhancing the training process (Hughes and Franks, 2004). Nevertheless, there is a lack of research that investigated game demands in elite players during official games, especially through the application of micro-technology. The use of data collected in games must be considered a priority when selecting training loads, especially when planning specific training drills (Svilar et al., 2018) that replicate the demands of a basketball game (e.g. 5 vs 5 . training games).

New micro-technologies (e.g., accelerometer, gyroscope, and magnetometer) can register a high amount of data, enabling practitioners to quantify training loads (Bucheit and Simpson, 2017). There are still not enough data to compare external training demands between basketball players (Stojanović et al., 2017). However, in the last year, steps are being taken in the study of basketball through the use of microtechnology (Vázquez-Guerrero et al., 2018). Due to the huge amount of data available per second of activity during a game, for describing player-dependent, game-related physical demands, some strategy is required. The principal component analysis (PCA) is a useful option to remove the redundancy in variables used to monitor loads (Weaving et al., 2014). Svilar et al. (2018) studied the training process of an elite team and concluded each postion had its particularity regarding acceleration, deceleration, jumps and change of direction. However, to date, limited

1 - Department of Education and Sport, University of the Basque Country (UPV/EHU), Vitoria-Gasteiz, Spain.

2 - Basketball Club Baskonia, Vitoria-Gasteiz, Spain. 
studies focused on basketball game demands and position-dependent characteristics of the elite basketball match-play using micro-technologies have been published (Montgomery et al., 2010; Vázquez-Guerrero et al., 2018; Puente et al., 2016).

Therefore, the purpose of the current study was to investigate the structure of interrelationships among the physical demands expressed in microtechnology variables and to determine how these parameters vary among different positions in elite basketball. Identifying position-dependent variables based on the inertial movement patterns of each playing position in elite basketball games could be useful for designing training strategies in order to prepare players to face specific demands of competition.

\section{Methods}

\section{Participants}

Seventeen elite male professional basketball players volunteered to participate in the study. Playing positions were: guards (age: $27.5 \pm 6$ years; body height: $188.0 \pm 1.0 \mathrm{~cm}$; body mass: $86.3 \pm 10.1 \mathrm{~kg}$; body fat: $9.6 \pm 0.7 \%$ ), forwards (age: $26.7 \pm 2.5$ years; body height: 193.9 $\pm 5.5 \mathrm{~cm}$; body mass: $92.9 \pm 7.5 \mathrm{~kg}$; body fat: $10.7 \pm$ $0.6 \%$ ) and centers (age: $23.1 \pm 2.0$ years; body height: $209.0 \pm 4.2 \mathrm{~cm}$; body mass: $107.3 \pm 6.8 \mathrm{~kg}$; body fat: $10.8 \pm 2.2 \%$ ). The weekly schedule consisted of 4 to 6 strength and team technicaltactical sessions, and one or two pre-season games in week 3, 4 and 5. The data were anonymized, and institutional approval was given for the study.

\section{Measures}

The external training load (eTL) included: Player Load (PL, using the formula from Barret et al., 2014), changes of direction (COD), jumps (JUMP), decelerations (DEC) and accelerations (ACC). The COD variable comprised two variables; tCOD as the total inertial movements registered in a rightward/leftward lateral vector, and; hCOD which was the total inertial movements registered in a rightward/leftward lateral vector within the high-intensity threshold $\left(>3 \mathrm{~m} \cdot \mathrm{s}^{-2}\right)$. The time interval during which acceleration is measured can significantly affect the data (Bucheit et al., 2017). The dwell time or minimum effort duration (MED) in the present study was set to $0.4 \mathrm{~s}$, since Varley et al. (2017) concluded that it was difficult to provide an appropriate MED with acceleration efforts. Jumps were registered as total jumps (tJUMP) and highintensity jumps (hJUMP, over $0.4 \mathrm{~m}$ ) (Spangler et al., 2018). The DEC and ACC variables were defined as inertial movements registered in forward deceleration and acceleration vectors, respectively. $\mathrm{tDEC}$ and $\mathrm{tACC}$ were described as the total amount of DEC and ACC, respectively, while hDEC and hACC referred only to the ones above the high-intensity threshold $\left(>3.5 \mathrm{~m} \cdot \mathrm{s}^{-2}\right)$. Furthermore, all aforementioned variables were assessed with respect to their frequency and normalized by duration (minutes of play) which have been previously used in elite basketball investigations (Svilar et al., 2018). Variables such as ACC/DEC (Varley et al., 2012) and COD (Meylan et al., 2016) have been previously investigated as part of micro-technology-derived data validity and reliability studies.

\section{Design and Procedures}

Elite-level basketball players were monitored for five match-days played during the pre-season period. Players were assigned to one of the three positional groups: guards (6), forwards (4), and centers (7). Five game observations were undertaken with a range of 518 quarter games per player. Quarter observations $(n=183)$ for each positional category were 78,37 and 68 for guards, forwards and centers, respectively. Games were based on basketball standard rules of competition with 4 quarters of 10 minutes, with 2 minutes of rest between quarters and 15 minutes between the second and third quarters.

Monitoring system T6 devices (Catapult ${ }^{\circledR}$, Canberra, Australia) were used to monitor the eTL. These recorded inertial movement analysis (IMA) based data through internal accelerometer, gyroscope and magnetometer sensors, with a sampling frequency of $100 \mathrm{~Hz}$. This kind of technology was previously confirmed as valid and reliable (Luteberget et al., 2018). After each game, all data were downloaded and processed with Openfield v1.14.0 software (Catapult ${ }^{\circledR}$, Canberra, Australia). Only data from the live period were selected and rest periods between quarters ( $2 \mathrm{~min})$, halves (15 $\mathrm{min})$ and timeouts were excluded from the analysis. Finally, the full data matrix was exported to IBM-SPSS Statistic software (IBM SPSS, Version 24.0. Armonk, NY: IBM Corp.) for statistical analysis. 


\section{Statistical Analysis}

Descriptive analyses were performed for all variables. The differences were assessed using Cohen's $d$ effect size (ES) (Cohen, 1988): trivial < 0.2 , small $=0.2<0.5$, moderate $=0.5<0.8$, and large $>0.8$. Principal Component Analysis (PCA) was used to extract the most important components. The Kaiser-Meyer-Olkin (KMO) values for the three different playing positions (guards, forwards and centers) were 0.72, 0.47 and 0.68 , respectively, showing that the dataset was suitable for PCA (Kaiser, 1960). In order to identify components that were not highly correlated, the PCA was applied with a VariMax rotation. For each extracted component, only the original variables that possessed a PC loading greater than 0.7 were retained for interpretation. The correlation among eTL variables was measured for each playing position. According to Hopkins (2000): trivial $=0-0.09$, small $=0.1-0.29$, moderate $=0.3-0.49$, large $=0.5-0.69$, very large $=$ $0.7-0.89$, nearly perfect $=0.9-0.99$, and perfect. The IBM-SPSS Statistic software version 24.0 (Armonk, NY: IBM Corp.) was used to conduct the analysis.

\section{Results}

The PLmin ranged from 10.5 to 12.1 arbitrary units (AU) for all positions (Figure 1). Guards presented the highest values $(12.1 \pm 2.0$ $\mathrm{AU} ; \mathrm{ES}=0.73$ vs. centers; $\mathrm{ES}=0.90$ vs. forwards), then there were forwards $(10.5 \pm 1.5 \mathrm{AU}$; ES $=0.12$ vs. center) and finally centers (10.7 $\pm 1.8 \mathrm{AU})$.

As it can be observed, out of the four eTL movements presented, the COD was the most frequent in a basketball game, followed by DEC, ACC and JUMP variables, respectively (Table 1).

Table 2 shows the PCA for the three playing positions and the total explained variance. For the three playing positions, PCs componentes explained $\approx 75 \%$ of the total variance, but with a different distribution of the external variables for each component. From the eight eTL metrics, the majority of eTL information ( $1^{\text {st }}$ PC: from 24 to $40 \%$ ) for the players' position could be explained by either tACC and hACC for centers and guards, or tCOD and hCOD for forwards and guards. The third PC was the same for the three playing positions and only forwards showed the fourth PC.

Table 1

Means and standard deviation (sd), and effect size (ES) of external training load measures according to the playing position.

\begin{tabular}{|c|c|c|c|c|c|c|c|c|c|}
\hline \multirow{2}{*}{$\begin{array}{l}\text { Variables } \\
(\mathrm{n} / \mathrm{min})\end{array}$} & \multicolumn{2}{|c|}{ Guards $(\mathrm{n}=78)$} & \multicolumn{2}{|c|}{ Forwards $(\mathrm{n}=37)$} & \multicolumn{2}{|c|}{ Centers $(n=68)$} & \multirow{2}{*}{$\begin{array}{l}\text { G vs. F } \\
\text { ES }\end{array}$} & \multirow{2}{*}{$\begin{array}{l}\text { G vs. C } \\
\text { ES }\end{array}$} & \multirow{2}{*}{$\begin{array}{l}\text { F vs. C } \\
\text { ES }\end{array}$} \\
\hline & Mean & sd & Mean & sd & Mean & sd & & & \\
\hline tACC & 2.1 & 0.7 & 1.8 & 0.6 & 2.6 & 0.9 & 0.46 & 0.62 & 1.05 \\
\hline hACC & 0.3 & 0.2 & 0.2 & 0.1 & 0.4 & 0.2 & 0.63 & 0.50 & 1.26 \\
\hline $\mathrm{tDEC}$ & 2.4 & 0.6 & 2.5 & 0.6 & 2.3 & 0.6 & 0.16 & 0.16 & 0.33 \\
\hline hDEC & 0.3 & 0.2 & 0.2 & 0.2 & 0.1 & 0.1 & 0.49 & 1.26 & 0.63 \\
\hline tCOD & 11.4 & 3.5 & 11.2 & 3.2 & 10.2 & 2.6 & 0.05 & 0.38 & 0.34 \\
\hline hCOD & 0.8 & 0.5 & 0.8 & 0.3 & 0.6 & 0.3 & 0.00 & 0.48 & 0.66 \\
\hline tJUMP & 0.9 & 0.5 & 1.3 & 0.4 & 1.2 & 0.5 & 0.88 & 0.59 & 0.22 \\
\hline hJUMP & 0.2 & 0.1 & 0.2 & 0.1 & 0.2 & 0.2 & 0.00 & 0.00 & 0.00 \\
\hline
\end{tabular}

Note: $t A C C$ is total forward acceleration, $h A C C$ is high intensity acceleration $\left(>3.5 \mathrm{~m} \cdot \mathrm{s}^{-2}\right), t D E C$ is total deceleration, hDEC is high intensity deceleration $\left(<-3.5 \mathrm{~m} \cdot \mathrm{s}^{-2}\right)$, tJUMP is total jumps, hJUMP is high intensity jumps (above $0.4 \mathrm{~m}$ ), tCOD is total rightward/leftward lateral movements, $h C O D$ is high intensity movements registered in a rightward/leftward lateral vector $\left(>3 \mathrm{~m} \cdot \mathrm{s}^{-2}\right)$. G: guards; F: forwards; C: centers. 
Table 2

Results of the Principal Components $(P C)$ analysis, showing the eigenvalue, percentage of variance explained (\% of V.), and the cumulative \% of variance explained (C.V.\%) by each PC for each playing position. Also showing the rotated load metrics component loadings for each PC extracted.

\begin{tabular}{|c|c|c|c|c|c|c|c|c|c|}
\hline \multirow{2}{*}{\multicolumn{2}{|c|}{ Playing position }} & \multicolumn{8}{|l|}{ PC } \\
\hline & & 1 & 2 & 3 & 4 & 5 & 6 & 7 & 8 \\
\hline \multirow{11}{*}{ 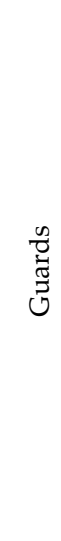 } & Eigenvalue & 3.21 & 1.51 & 1.06 & .66 & .54 & .38 & .33 & .28 \\
\hline & $\%$ of $\mathrm{V}$ & 40.23 & 18.93 & 13.27 & 8.30 & 6.76 & 4.82 & 4.12 & 3.53 \\
\hline & C. V. $\%$ & 40.23 & 59.16 & 72.44 & 80.74 & 87.51 & 92.33 & 96.46 & 100.00 \\
\hline & tACC & .75 & .32 & .08 & & & & & \\
\hline & hACC & .75 & -.09 & .09 & & & & & \\
\hline & $\mathrm{tDEC}$ & .00 & .30 & .78 & & & & & \\
\hline & hDEC & .15 & -.04 & .86 & & & & & \\
\hline & tCOD & .85 & -.03 & .08 & & & & & \\
\hline & hCOD & .81 & .19 & .01 & & & & & \\
\hline & tJUMP & .50 & .67 & .14 & & & & & \\
\hline & hJUMP & -.05 & .92 & .16 & & & & & \\
\hline \multirow{11}{*}{$\begin{array}{l}0 \\
0 \\
0 \\
3 \\
0 \\
0 \\
0\end{array}$} & Eigenvalue & 1.94 & 1.75 & 1.25 & 1.07 & .75 & .59 & .34 & .27 \\
\hline & $\%$ of $\mathrm{V}$ & 24.26 & 21.96 & 15.67 & 13.47 & 9.48 & 7.41 & 4.32 & 3.39 \\
\hline & C. V. $\%$ & 24.26 & 46.23 & 61.90 & 75.38 & 84.86 & 92.27 & 96.60 & 100.00 \\
\hline & tACC & .01 & .89 & .10 & -.03 & & & & \\
\hline & hACC & -.22 & .69 & -.09 & .20 & & & & \\
\hline & $\mathrm{tDEC}$ & .31 & .45 & .69 & -.22 & & & & \\
\hline & hDEC & -.19 & -.10 & .88 & .14 & & & & \\
\hline & tCOD & .86 & -.17 & -.04 & .05 & & & & \\
\hline & hCOD & .89 & .00 & .02 & -.06 & & & & \\
\hline & tJUMP & .29 & -.07 & .41 & .61 & & & & \\
\hline & hJUMP & -.13 & .14 & -.08 & .82 & & & & \\
\hline \multirow{11}{*}{ U⿺辶寸 } & Eigenvalue & 2.86 & 1.48 & 1.00 & .84 & .59 & .50 & .42 & .28 \\
\hline & $\%$ of $\mathrm{V}$ & 35.79 & 18.60 & 12.53 & 10.50 & 7.40 & 6.33 & 5.25 & 3.55 \\
\hline & C. V. $\%$ & 35.79 & 54.40 & 66.93 & 77.44 & 84.85 & 91.18 & 96.44 & 100.00 \\
\hline & tACC & .83 & .04 & .11 & & & & & \\
\hline & hACC & .72 & -.09 & .28 & & & & & \\
\hline & $\mathrm{tDEC}$ & .44 & .59 & .03 & & & & & \\
\hline & hDEC & .11 & .16 & .90 & & & & & \\
\hline & tCOD & .65 & .28 & -.34 & & & & & \\
\hline & hCOD & .65 & .19 & .00 & & & & & \\
\hline & tJUMP & .24 & .82 & .09 & & & & & \\
\hline & hJUMP & -.16 & .85 & .05 & & & & & \\
\hline
\end{tabular}

Note: $t A C C$ is total forward acceleration, $h A C C$ is high-intensity acceleration $\left(>3.5 \mathrm{~m} \cdot \mathrm{s}^{-2}\right), t D E C$ is total deceleration, $h D E C$ is high-intensity deceleration $\left(<-3.5 \mathrm{~m} \cdot \mathrm{s}^{-2}\right)$, tJUMP is total jumps, hJUMP is highintensity jumps (above $0.4 \mathrm{~m}$ ), tCOD is total rightward/leftward lateral movements, $h C O D$ is highintensity movements registered in a rightward/leftward lateral vector $\left(>3 \mathrm{~m} \cdot \mathrm{s}^{-2}\right)$. 
Table 3

Pearson correlations among external load variables for each playing position.

\begin{tabular}{|c|c|c|c|c|c|c|c|c|c|}
\hline & & tJUMP & hJUMP & tACC & hACC & $\mathrm{tDEC}$ & hDEC & $\mathrm{tCOD}$ & hCOD \\
\hline \multirow{8}{*}{$\begin{array}{l}\frac{n}{0} \\
\tilde{\Xi} \\
\tilde{U}\end{array}$} & PLmin & $.294^{* *}$ & .211 & $.535^{* *}$ & $.305^{* *}$ & $.379^{* *}$ & $.282^{*}$ & $.252^{*}$ & $.277^{*}$ \\
\hline & tJUMP & & $.491^{* *}$ & $.507^{* *}$ & $.287^{*}$ & .198 & $.271^{*}$ & $.382^{* *}$ & $.527^{* *}$ \\
\hline & hJUMP & & & $.230^{*}$ & -.016 & $.335^{* *}$ & .072 & -.024 & .103 \\
\hline & tACC & & & & $.509^{* *}$ & .223 & .136 & $.564^{* *}$ & $.563^{* *}$ \\
\hline & hACC & & & & & .082 & .150 & $.515^{* *}$ & $.450^{* *}$ \\
\hline & $\mathrm{tDEC}$ & & & & & & $.416^{* *}$ & .097 & .086 \\
\hline & $\mathrm{hDEC}$ & & & & & & & .174 & .156 \\
\hline & $\mathrm{tCOD}$ & & & & & & & & $.652^{* *}$ \\
\hline \multirow{8}{*}{$\begin{array}{l}\text { o } \\
\text { J } \\
3 \\
0 \\
0 \\
0\end{array}$} & PLmin & .204 & -.059 & $.341^{*}$ & -.108 & $.512^{* *}$ & .005 & $.427^{* *}$ & $.557^{* *}$ \\
\hline & tJUMP & & .153 & -.013 & .021 & .156 & .254 & .198 & .158 \\
\hline & hJUMP & & & .117 & .123 & -.109 & .093 & .100 & -.116 \\
\hline & tACC & & & & $.382^{*}$ & $.423^{* *}$ & -.017 & -.194 & .026 \\
\hline & hACC & & & & & .077 & .001 & -.178 & -.232 \\
\hline & $\mathrm{tDEC}$ & & & & & & $.385^{*}$ & .146 & .264 \\
\hline & $\mathrm{hDEC}$ & & & & & & & -.102 & -.089 \\
\hline & tCOD & & & & & & & & $.628^{* *}$ \\
\hline \multirow{8}{*}{ 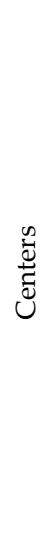 } & PLmin & $.423^{* *}$ & .106 & $.472^{* *}$ & $.246^{*}$ & $.484^{* *}$ & .009 & $.854^{* *}$ & $.491^{* *}$ \\
\hline & tJUMP & & $.540^{* *}$ & $.315^{* *}$ & .157 & $.516^{* *}$ & .190 & $.293^{*}$ & .231 \\
\hline & hJUMP & & & -.080 & -.062 & $.292^{*}$ & .124 & .094 & .126 \\
\hline & tACC & & & & $.556^{* *}$ & $.389^{* *}$ & .124 & $.361^{* *}$ & $.439^{* *}$ \\
\hline & hACC & & & & & $.266^{*}$ & .175 & $.272^{*}$ & $.278^{*}$ \\
\hline & tDEC & & & & & & .146 & $.358^{* *}$ & .225 \\
\hline & $\mathrm{hDEC}$ & & & & & & & .009 & .183 \\
\hline & $\mathrm{tCOD}$ & & & & & & & & $.470^{* *}$ \\
\hline
\end{tabular}

Note: $t A C C$ is total forward acceleration, $h A C C$ is high-intensity acceleration $\left(>3.5 \mathrm{~m} \cdot \mathrm{s}^{-2}\right), t D E C$ is total deceleration, $h D E C$ is high-intensity deceleration $\left(<-3.5 \mathrm{~m} \cdot \mathrm{s}^{-2}\right)$, tJUMP is total jumps, hJUMP is high intensity jumps (above $0.4 \mathrm{~m}$ ), tCOD is total rightward/leftward lateral movements, $h C O D$ is high intensity movements registered in a rightward/leftward lateral vector $\left(>3 \mathrm{~m} \cdot \mathrm{s}^{-2}\right)$, PLmin is player load per minute. In bold large or very large qualitative correlation descriptor. Correlations had a significant value at ${ }^{*} p<0.05$ and ${ }^{* *} p<0.01$ level. 


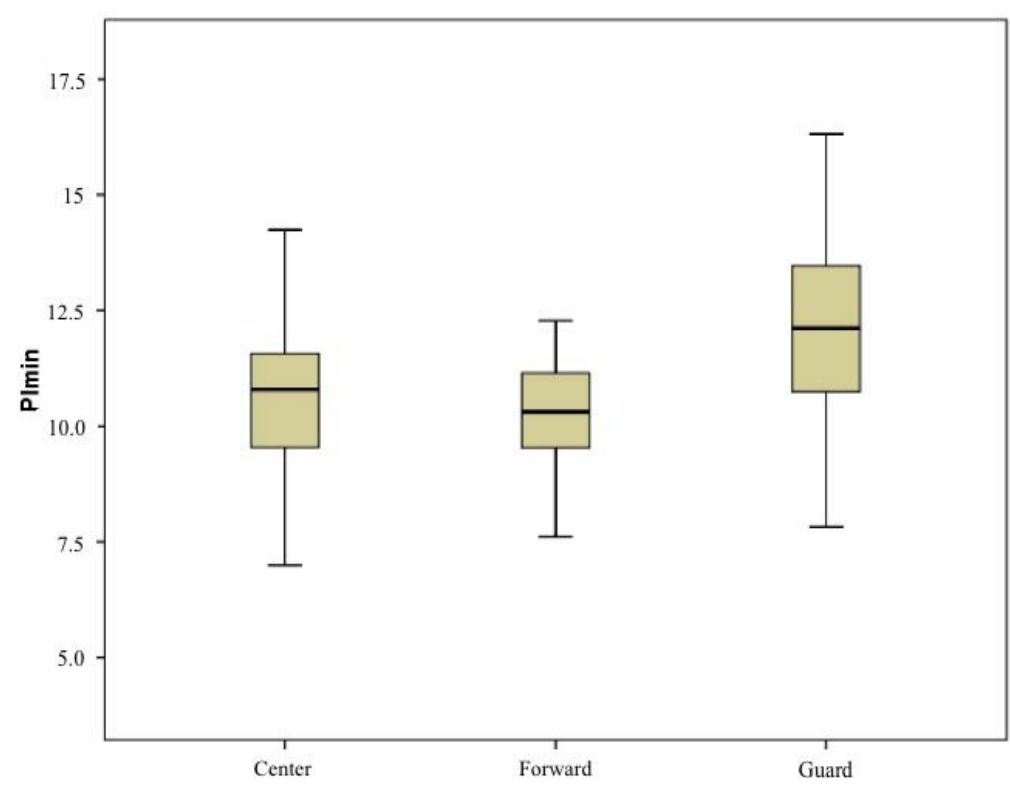

$\mathrm{X}$

\section{Figure 1}

Player load per minute (PLmin) per position during match-play.
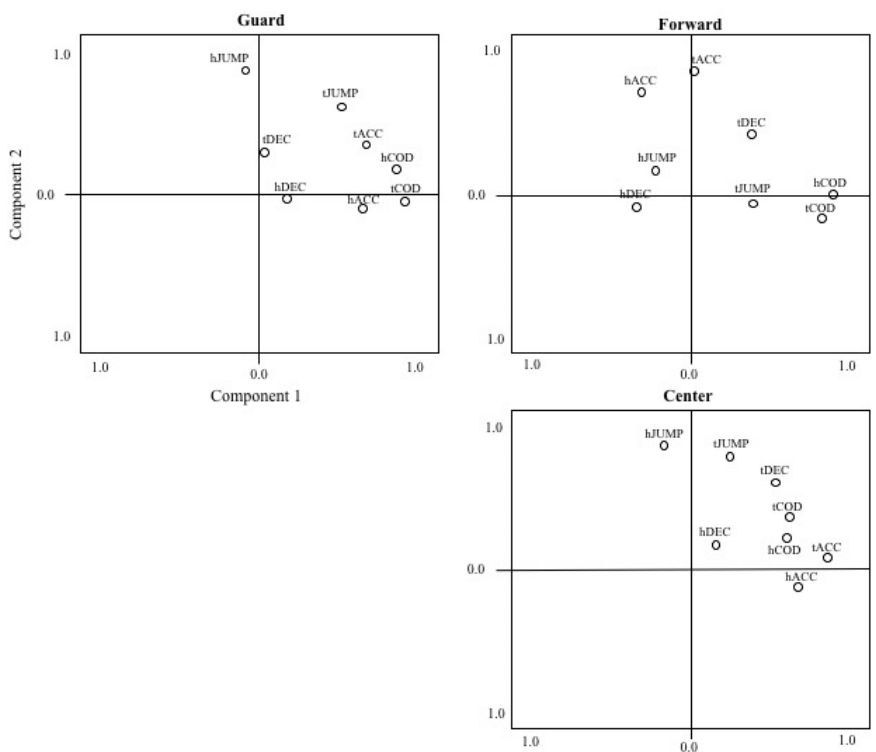

Figure 2

Two principal rotated components for the three playing positions

Note: $t A C C$ is total forward acceleration, $h A C C$ is high-intensity acceleration $\left(>3.5 \mathrm{~m} \cdot \mathrm{s}^{-2}\right), t D E C$ is total deceleration, $h D E C$ is high-intensity deceleration $\left(<-3.5 \mathrm{~m} \cdot \mathrm{s}^{-2}\right)$, tJUMP is total jumps, hJUMP is highintensity jumps (above $0.4 \mathrm{~m}$ ), tCOD is total rightward/leftward lateral movements, $h C O D$ is highintensity movements registered in a rightward/leftward lateral vector $\left(>3 \mathrm{~m} \cdot \mathrm{s}^{-2}\right)$. 
Figure 2 illustrates a rotated PC for each playing position. Only the two main factors were plotted to visually represent playing position differences.

Finally, Pearson correlations between eTL variables for each playing position are presented in Table 3. There was a strong and positive correlation between COD variables $(r=0.652, p<$ $0.01)$ and tACC with tCOD $(r=0.564, p<0.01)$ for guards. Similarly to guards, forwards showed the higest correlation between COD variables, as well as between hCOD and PLmin $(r=0.557, p<0.001)$. For centers, tCOD with PLmin $(r=0.854, p<0.01)$ and ACC variables $(r=0.556, p<0.01)$ presented the highest correlations.

\section{Discussion}

The main finding of the present study showed a different weight of eTL variables for the three different playing positions defined during match-play, based on the identification of a structure with three or four PCs summarizing several physical demands.

Since PL calculation takes into account the volume and intensity of movements (Bredt et al., 2019), PLmin is higher in guards than in the other two positions. Recent research corroborates this result (Vázquez-Guerrero et al., 2018). However, values differ between studies due to divergence in the calculation of the Player Load.

More sprints per minute and a number of ACC/DEC ratio per minute were performed by guards during the $20 \mathrm{~min}$ non-official games recorded with GPS technology (Puente et al., 2016). Usually, coaches ask centers to perform rebounding, screening and short-middle range shooting. Actions like boxing out and screening are static efforts that are not likely to be detected by accelerometry (Schelling and Torres-Ronda, 2016) and consequently are not considered for PL calculation. However, centers have higher values of PLmin than forwards, although this position is usually characterized by playing in more reduced areas of the court and covering less total distance and high-intensity distance (Puente et al., 2016). This study showed that PL, regardless of the playing position, is a variable sensitive to all specific types of movements (ACC, DEC or JUMP). Due to the aforementioned, the results in this study support the fact that PL is a good indicator of the external load (Bredt et al.,
2019). Previous findings reported no differences in the relative frequency of movements (TorresRonda et al., 2016). However, regarding the intensity of movements, Svilar et al. (2018) found significant difference for hACC between matchplay and training games (match play $>$ training game), which was explained by mental factors that may motivate players to perform at higher intensity when playing against real opponents. The correlations of the PLmin with different variables depending on the playing position showed that, although there were practically no differences in the PLmin between players, guards' PLmin correlated with tACC, forwards' with tDEC and hCOD and finally centers' with tCOD.

ACC dimension was expressed as the first component for guards and centers, while tACC appeared in the second component for forwards. The forward position was the playing position that had particularly different statistical results compared to guards and centers (e.g., four principal components, very low correlations, or low KMO value). It seems that this playing position has the greatest performance variability and on the court forwards play a mixed role between centers and guards, what makes the interpretation of game-based demands difficult for this specific playing role. Furthermore, for guards, ACC correlates with COD variables. The anthropometric profile is known to be the main factor that defines court positions in basketball. Guards are smaller with less body mass, which allows them to accelerate faster than their teammates of a higher stature (Torres-Ronda et al., 2016). Moreover, as previous research has pointed (Hulka et al., 2013), smaller players have a greater playing zone, covering more distance and making it easier to perform a higher number of accelerations and achieve greater movement velocity. This fact could explain why ACC and COD variables are the first components in their profile. Additionally, the physical characteristics of the centers in modern basketball are changing. They now have a much greater coordination capacity and are capable of making fast and accurate movements in both small spaces and open court. Besides, they show the worst results among the three playing positions in explosive tasks (Pehar et al., 2017).

The variables involving COD seem to play an important role in basketball physical 
performance because it appears as the first component for both guards and forwards. Basketball is an indoor team sport with a small court, enabling players to use the court in a horizontal way inside the three-point line with small movements looking for the free space to shoot, dribble or pass. In contrast with the training sessions where tCOD seemed to be the first component for all positions (Svilar et al., 2018), the profile of centers did not show it during games. Training drills during practice may demand different physical requirements from centers than what actually occurs in games.

As a previous study has described, centers are the players who perform the highest number of jumps during the game (Abdelkrin et al., 2007). In this study, hJUMP was representative for centers and guards as the second component, while for forwards it was the forth component. In addition, tJUMP was only representative for centers as the second component. This finding, together with the training data (Svilar et al., 2018), could indicate that jumps are a movement pattern that is not as frequent in basketball as it was thought to be, especially when compared with accelerations and changes of direction.

Decelerations only appeared as the third component for all playing positions, contrary to the previous training data where DEC appeared as the first component for forwards and centers (Svilar et al., 2018). This variable may not be as significant as the first component, but still plays an important role, which is why strength and conditioning coaches should take it into account in order to teach good deceleration technique independent of the player's position. Regarding the relationship between decelerations and other variables, the strongest relation was found with
PLmin for forwards and tJUMP for centers. The present study showed that hCOD for guards and forwards as well as hACC for centers could be interesting variables to assess the intensity of the load for these three positions. Nevertheless, a combination of external training load variables is required to describe positional demands in elite basketball games. This study presents a new external load profiling that can be used to describe a basketball game considering three playing positions. Future research should focus more on the implementation of objective microtechnology and the analysis of external load variables in elite basketball competition and training.

The results of this study should be interpreted with caution. First of all, due to a small sample size in the current study (one team) only five games were recorded. Secondly, a low value of $\mathrm{KMO}$, the need for the fourth PC in their profile and the small number of significant correlations found can be explained by the low (n $=4$ ) number of forwards included in the study. In the future, it could be more reliable to include a greater number of players and games, in order to avoid the influence of contextual variables such as the location (home/away), rival quality, type of competition, etc.

To conclude, COD and ACC variables can define the physical profile during games for elite basketball players. Furthermore, JUMP variable seems to play a secondary role in centers and guards. These results should help coaches to manage the load monitoring process, focusing on variables which better describe individual profiles of elite players for game demands.

\section{Acknowledgements}

The authors would like to thank the coaching staff and players of the basketball club Saski Baskonia S.A.D. for their participation in this study. Furthermore, we gratefully acknowledge the support of the Spanish government project "The role of physical activity and sport in the promotion of healthy lifestyle habits: the evaluation of sport behavior using non-intrusive methods" during the period 2016-2018 [Grant number DEP2015-66069-P, MINECO/FEDER, UE].

\section{References}

Barret S, Midgley A, Lovell R. PlayerLoad: Reliability, convergent validity, and influence of unit position during treadmill running. Int J Sport Physiol, 2014; 9: 945-952

Bredt S, Chagas M, Peixoto G, Menzel H, Andrade A. Understanding Player Load: Meanings and Limitations. J Hum Kinet, 2019; doi: 10.2478/hukin-2019-0072 
Buchheit M, Simpson BM. Player tracking technology: Half-Full or half-empty glass? Int J Sport Physiol, 2017; 12: $35-41$

Cohen J. Statistical power analysis for the behavioral sciences (2nd ed.). Hillsdale, NJ: Lawrence Earlbaum Associates; 1988

Hopkins WG. A new view of statistics. Internet Society for Sport Science. http://www.sportsci.org/resource/stats/, 2000

Hughes M, Franks IM. Notational analysis of sport. Systems for better coaching and performance in sport. London: Routledge; 2004

Hulin BT, Gabbett TJ, Lawson DW, Caputi P, Sampson JA. The acute: chronic workload ratio predicts injury: high chronic workload may decrease injury risk in elite rugby league players. $\mathrm{Br} J$ Sports Med, 2015; 50: 231-236

Hulka K, Cuberek R, Belka J. Heart rate and time-motion analyses in top junior players during basketball matches. Acta Gymnica, 2013; 43: 27-35

Kaiser HF. The application of electronic computers to factor analysis. Educ Psychol Meas, 1960; 20: 141-151

Luteberget LS, Spencer M, Gilgien M. Validity of the catapult clearSky T6 local positioning system for team sports specific drills, in indoor conditions. Front Physiol, 2018; 9: 115

Meylan CM, Trewin J, McKean K. Quantifying explosive actions in international woman's soccer. Int J Sport Physiol, 2016; 12: 310-315

Montgomery PG, Pyne DB, Minahan CL. The physical and physiological demands of basketball training and competition. Int J Sports Physiol, 2010; 5: 75-86

Pehar M, Sekulic D, Sisic N, Spasic M, Uljevic O, Krolo A, Milanovic Z, Sattler T. Evaluation of different jumping tests in defining position-specific and performance-level differences in high level basketball players. Biol Sport, 2017; 34: 263-272

Puente C, Abián-Vicén J, Areces F, López R, Del Coso J. Physical and physiological demands of experienced male basketball players during a competitive game. J Strength Cond Res, 2017; 31: 956-962

Scanlan AT, Dascombe BJ, Reaburn PA. Comparison of the activity demands of elite and sub-elite Australian men's basketball competition. J Sports Sci, 2011; 29: 1153-60]

Scanlan AT, Dascombe BJ, Reaburn PA, Dalbo VJ. The physiological and activity demands experienced by Australian female basketball players during competition. J Sci Med Sport, 2012; 15: 341-347

Schelling X, Torres-Ronda L. Accelerometer load profiles for basketball-specific drills in elite players. J Sports Sci Med, 2016; 15: 585-591

Spangler R, Rantalainen T, Gastin PB, Wundersitz D. Inertial sensors are a valid tool to detect and consistently quantify jumping. Int J Sports Med, 2018; 39: 802-808

Stojanović E, Stojiljković N, Scanlan AT, Dalbo VJ, Berkelmans DM, Milanović Z. The activity demands and physiological responses encountered during basketball match-play: A Systematic Review. Sports Med, 2017; 48: 111-135

Svilar L, Castellano J, Jukic I, Casamichana D. Positional differences in elite basketball: Selecting appropriate training-load measures. Int J Sport Physiol, 2018; 13: 947-952

Svilar L, Castellano J, Jukic I. Comparison of 5vs5 training games and match-play using micro-sensor technology in elite basketball. J Strength Cond Res, 2018; [Epub ahead of print]

Svilar L, Castellano J, Jukić I. Load monitoring system in top-level basketball team: relationship between external and internal training load. Kinesiology, 2018; 50: 25-33

Torres-Ronda L, Ric A, Llabres-Torres I, De las Heras B, Schelling X. Position-dependent cardiovascular response and time-motion analysis during training drills and friendly matches in elite male basketball players. J Strength Cond Res, 2016; 30: 60-70

Varley MC, Fairweather IH, Aughey RJ. Validity and reliability of GPS for measuring instantaneous velocity during acceleration, deceleration, and constant motion. J Sports Sci, 2012; 30: 121-127

Varley MC, Jaspers A, Helsen WF, Malone JJ. Methodological considerations when quantifying highintensity efforts in team sport using global positioning system technology. Int J Sport Physiol, 2017; 12: $1-25$

Vazquez-Guerrero J, Reche X, Cos F, Casamichana D, Sampaio J. Changes in external load when modifying rules of 5-on-5 scrimmage situations in elite basketball. J Strength Cond Res, 2018; [Epub ahead of print] 
Vázquez-Guerrero J, Suarez-Arrones L, Casamichana D, Rodas G. Comparing external total load, acceleration and deceleration outputs in elite basketball players across positions during match play. Kinesiol, 2018; 50(2): 228-234

Weaving D, Marshall P, Earle K, Nevill A, Abt GA. Combination of internal and external training load measures explains the greatest proportion of variance in certain training modes in professional rugby league. Int J Sport Physiol, 2014; 9: 905-12

Ziv G, Lidor R. Physical attributes, physiological characteristics, on-court performances and nutritional strategies of female and male basketball players. Sports Med, 2009; 39: 547-568

\section{Corresponding author:}

\section{Hugo Salazar}

Department of Education and Sport, University of the Basque Country (UPV/EHU), Vitoria-Gasteiz (Spain) Phone number: 0034638122383

E-mail: hsalazar002@gmail.com 